\title{
Hydration of an amphiphilic excipient, Gelucire 44/14
}

\author{
A. Svensson ${ }^{\#}$, C. Neves* and B. Cabane ${ }^{+}$ \\ \# Department of Physical Chemistry 1, Chemical Center, University of Lund, POB 124, \\ S221 00 Lund, Sweden \\ * Aventis, Department of Pharmaceutical Sciences, Paris Research Center, 94400 Vitry-sur- \\ Seine, France \\ + Laboratoire PMMH, CNRS UMR 7636, ESPCI, 10 rue Vauquelin, 75231 Paris Cedex 05, \\ France
}

\section{Abstract}

The hydration behavior of an amphiphilic excipient, Gelucire 44/14, has been investigated. Two types of hydration processes were studied: one with increasing humidity to investigate the conditions during storage, and one with increasing water contents to study the behavior during dissolution. In addition, the main components of the excipient were investigated separately. These were polyethylene glycol (PEG), PEG monolaurate and PEG dilaurate (PEG esters), trilaurin (glyceride) and glycerol. The water uptake of Gelucire 44/14 at humidity ratios less than $60 \% \mathrm{RH}$ was very low (about $1 \mathrm{wt} \%$ ), which was attributed to the dissolution of the most hydrophilic component, glycerol. The water uptake increased substantially above $70 \% \mathrm{RH}$ as PEG started to dissolve, followed by the PEG esters. It was concluded that each component equilibrates separately with the aqueous solution, which itself is in equilibrium with the humid air. Hence, a liquid phase can form between the crystals with a chemical potential decided by the humidity ratio. The water uptake of Gelucire 44/14 could be described as a sum of the uptake of the individual components, weighted according to their relative amounts in the mixture. Phase maps of the Gelucire 44/14 and its components at different water contents were constructed. Dry Gelucire 44/14 contains lamellar crystals of mainly PEG and PEG esters which melt at $44{ }^{\circ} \mathrm{C}$. The crystals do not swell at increasing humidity, but dissolve above $75 \% \mathrm{RH}$ at a water content of $5 \mathrm{wt} \%$ in the excipient. At increasing water contents Gelucire 44/14 forms white gels composed of hexagonal and lamellar mesophases dispersed in a continuous liquid phase. These liquid crystalline phases dissolve at $35{ }^{\circ} \mathrm{C}$, i.e. below physiological temperatures. A dramatic viscosity maximum was observed in the lamellar region at $50 \mathrm{wt} \%$ water, which may be attributed to the formation of networks of PEG esters. The pure PEG esters were found to form cubic mesophases at $50 \mathrm{wt} \%$ water. The instruments used in this study were Dynamic Vapor Sorption (DVS), Thermal Gravimetric Analysis (TGA), Differential Scanning Calorimetry (DSC), Smalland Wide Angle X-ray Scattering (SWAXS) and Optical Microscopy. 


\section{Keywords}

Gelucire 44/14; phase diagram; hydration; DVS; SWAX

\section{Introduction}

Gelucires are a group of amphiphilic excipients which have been widely studied as controlled release matrices (Mouricout et al., 1990). The incorporation of drugs into Gelucires has been reported to increase the dissolution rate of poorly soluble drugs, often leading to improved drug bioavailability (Gines et al., 1995; Damian et al., 2000; Perissutti et al., 2000; Gupta et al., 2001). One compound from this group is Gelucire 44/14. This amphiphilic excipient has a Hydrophilic-Lipophilic Balance of 14 and a melting temperature of $44{ }^{\circ} \mathrm{C}$, hence its name (Roussin et al., 1997).

In pharmaceutical applications, it is important to know how the excipient interacts with the drug, and how the mixture behaves during manufacturing, storage as well as during administration. These behaviours depend on the effects of temperature and hydration on the physical state of the excipient. For instance, dissolution or dispersion of a drug in the excipient can be modified according to water content; conversely, the synthesized drug may be hydrated, and this water may alter the physical state of the excipient (Damian et al., 2002; Sutananta et al., 1994a; Jeanmaire-Wolf et al., 1990). The excipient-drug mixture may then be stored at a certain relative humidity, and moisture form the air may have an effect on its physical state. Finally, during administration, the mixture will be immersed in body fluids, and it is important to know what are the pathways for dissolution in this aqueous medium.

There have been a few studies of the thermal behaviour of dry Gelucires (Craig et al., 1991; Sutananta et al., 1994b), but, to our knowledge, no systematic study of their hydration behaviour. The aim of this project was to investigate the behaviour of the Gelucire 44/14 when exposed to humidity and water, at ambient and physiological temperatures. We studied two hydration processes, corresponding respectively to the conditions encountered during storage and during dissolution of excipients. During storage, the excipients may be kept in equilibrium with an atmosphere of constant relative humidity $(\mathrm{RH})$. Accordingly, we have measured the mass of water absorbed as a function of RH, through DVS experiments. During dissolution, the excipients are mixed with liquid water. Accordingly, we have also investigated the state of Gelucire 44/14 mixed with known amounts of water, or individual components mixed with known amounts of water. The nature of the phases (crystal, liquid crystal or isotropic solution) that are formed at selected compositions and different temperatures was determined, and their structural parameters were also measured. 
Since Gelucire itself is a mixture, its hydration behaviour is determined by the behaviours of its components, and their interactions. Thus, in addition to Gelucire 44/14, we also investigated the pure components separately in order to distinguish their specific effects when mixed together. Gelucire 44/14 is composed of polyethylene glycol 33 (PEG 33), PEG mono- and diesters of fatty acids, glycerides and a small amount of glycerol. The most common fatty acid chain in the mixture is laurate. Consequently, we decided to investigate the following chemicals: PEG 33, PEG monolaurate, PEG dilaurate and Trilaurin (triglyceride with laurate chains). By using thermal analysis, microscopy and X-ray diffraction, we investigated the phase behaviour of Gelucire 44/14 and compared it with the phase behaviour of the simple components. In the end, we found that the hydration behaviour of Gelucire 44/14 can be explained by the behaviours of its components.

\section{Materials and methods}

Gelucire 44/14 (Gattefosse s.a) is produced by the reaction of hydrogenated palm kernel oil and polyethylene glycol, PEG $33(1500 \mathrm{~g} / \mathrm{moL})$. It contains mostly fatty acids of the lauric type (i.e. C12 chains) of which more than $80 \%$ are saturated. The final composition in the Gelucire 44/14 is $72 \mathrm{wt} \%$ PEG esters, $20 \mathrm{wt} \%$ glycerides, $8 \mathrm{wt} \%$ pure PEG and 2\% glycerol. The PEG esters are composed by PEG mono- and diesters and the glycerides by mono-, di or triglycerides. Laurate acid chains are the most common type with 40-50\%. The most common chemicals in the Gelucire 44/14 mixture are PEG, PEG mono- and dilaurate (the PEG esters), mono-, di and trilaurates (the glycerides). PEG 33 and trilaurin (triglyceride with laurate chains) were purchased from Sigma. PEG monolaurate and PEG dilaurate were produced by organic synthesis.

\section{PEG ester synthesis}

$5 \mathrm{~g}$ PEG $33(0,0035 \mathrm{~mol})$ was dissolved in $25 \mathrm{ml}$ ethanol free dichloromethane in a flask. 0,5 ml triethylamin $(0,0035$ mole $)$ was added to buffer the reaction medium. The flask was put in an ice bath $\left(0{ }^{\circ} \mathrm{C}\right)$. The reaction started by the addition of $0,8 \mathrm{ml}$ lauryl chloride $(0,0035$ mole $)$. After 2 hours, the mixture contained the products PEG 33, PEG monolaurate and PEG dilaurate. Separation of the products was made by column chromatography on silica gel with a solvent containing dichloromethane:methanol with the proportions 9:1. Finally, the products were washed in cyclohexan to take away excess reactants. The structures of the PEG esters were confirmed by nuclear magnetic resonance, mass- and infrared spectrometry.

\section{Preparation of samples at different humidities}

Samples of Gelucire 44/14 were exposed to humidity ratios 0-97 \% RH, in order to investigate the humidity effects on the properties of the excipient. The samples were equilibrated in 
desiccators containing different saturated salt solutions: silica gel $(0 \% \mathrm{RH}), \mathrm{Mg}\left(\mathrm{NO}_{3}\right)_{2}$ (50\%RH), $\mathrm{CuCl}_{2}(68 \% \mathrm{RH}), \mathrm{NaCl}(75 \% \mathrm{RH}), \mathrm{KCl}(84 \% \mathrm{RH})$ and $\mathrm{K}_{2} \mathrm{SO}_{4}(97 \% \mathrm{RH})$.

\section{Preparation of samples with high water contents}

To investigate the phase behaviour at high water contents, samples were prepared by mixing directly with water. Samples with weight ratios of component and water from 10/90 to 75/25 were prepared. The component and water were weighed in small glass containers with a scale and sealed with a cap. The samples were mixed by hand and using an ultra sonic water bath. They were left to equilibrate for several weeks before investigation.

\section{Dynamic vapour sorption (DVS)}

The dynamic water sorption experiments were made with a DVS thermo-hygro-gravimetric balance under constant nitrogen sweeping and constant temperature $\left(25^{\circ} \mathrm{C}\right)$. The sample (assay sample of about $10 \mathrm{mg}$ ) was placed in a glass capsule. It was exposed to a series of relative humidity during a specific time interval at which the absorption of water is measured in weight. The steps are in $10 \% \mathrm{RH}$. The maximum time at each relative humidity is six hours. If equilibrium is reached within this time limit, i.e. the derivative of weight vs. time is $<0.02$, the measurement automatically moves on to the next humidity.

\section{Small and Wide Angle X-ray Scattering (SWAXS)}

To determine the presence of structures in the samples, X-ray diffraction at small and wide angles was used. The SWAXS measurements were performed with a Kratky compact small and wide angle system equipped with a linear collimation system and two position sensitive detectors (Hecus M Braun, Austria). Each detector contains 1024 channels of width $54.0 \mu \mathrm{m}$. A monochromator with a nickel filter was used to select the $\mathrm{Cu}-\mathrm{K}$ radiation $(\lambda=1.541 \AA)$ provided by the generator. The generator, a Seiffert ID-3003 X-ray, was operating at $50 \mathrm{kV}$ and $40 \mathrm{~mA}$. The sample was enclosed in a steel sample holder with mica windows. The distance between the sample and the detector was $279 \mathrm{~nm}$. The measurements were made at room temperature $\left(20^{\circ} \mathrm{C}\right)$ unless measurement with temperature increase $\left(20-60^{\circ} \mathrm{C}\right)$ was performed.

\section{Thermal Gravimetric Analysis (TGA)}

A sample with a mass between 1.5 and $4 \mathrm{mg}$ was deposited in an open 75-_L aluminum crucible. The sample was heated from $20^{\circ} \mathrm{C}$ to $250^{\circ} \mathrm{C}$ at a rate of $1^{\circ} \mathrm{C} / \mathrm{min}$. The analysis was carried out in a nitrogen stream. 


\section{Results}

In this section, the physical state and the hydration of Gelucire 44/14 are compared with those of the pure components. The aim is to find out how the excipient takes up water. Two alternative behaviours may take place : (a) the different components in the excipient are segregated, and they take up water independently ; (b) the components in the excipient are associated, forming solid or liquid solutions which take up water more readily (or less readily) than the segregated mixtures. It is also possible that the hydration process starts with the former behaviour (hydration of separated components) and then changes to the latter one through dissolution of 2 of more components in the same aqueous solution.

\section{General view of the hydration processes}

A general overview of the hydration processes is given by the water sorption isotherms measured through DVS experiments (Figure 1). Each isotherm shows the relative change of mass according to $\mathrm{RH}$ valued. Of particular interest are $\mathrm{RH}$ values around $50 \%$, which correspond to usual storage conditions. At $\mathrm{RH}=70 \%$ and above, the samples did not reach equilibrium hydration within the time of the DVS experiments. In this range, the DVS experiments were supplemented by TGA experiments where the samples were equilibrated against water vapour from saturated salt solutions. The differences between both sets of results were found to be small, and well within experimental errors. Moreover, the hydrations measured through both experiments for PEG 33, up to $\mathrm{RH}=90 \%$, are in good agreement with the values determined through osmotic pressure experiments by Rand and coworkers [yyy] (Figure 2). Since osmotic stress experiments yield true equilibrium hydrations, we may conclude that both DVS and TGA results gave equilibrium hydrations up to $\mathrm{RH}=90 \%$.

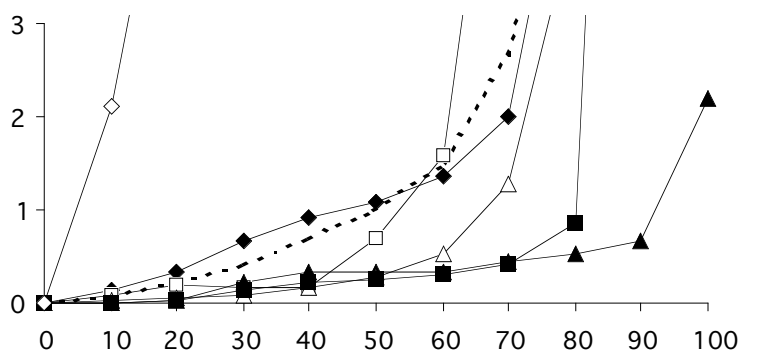




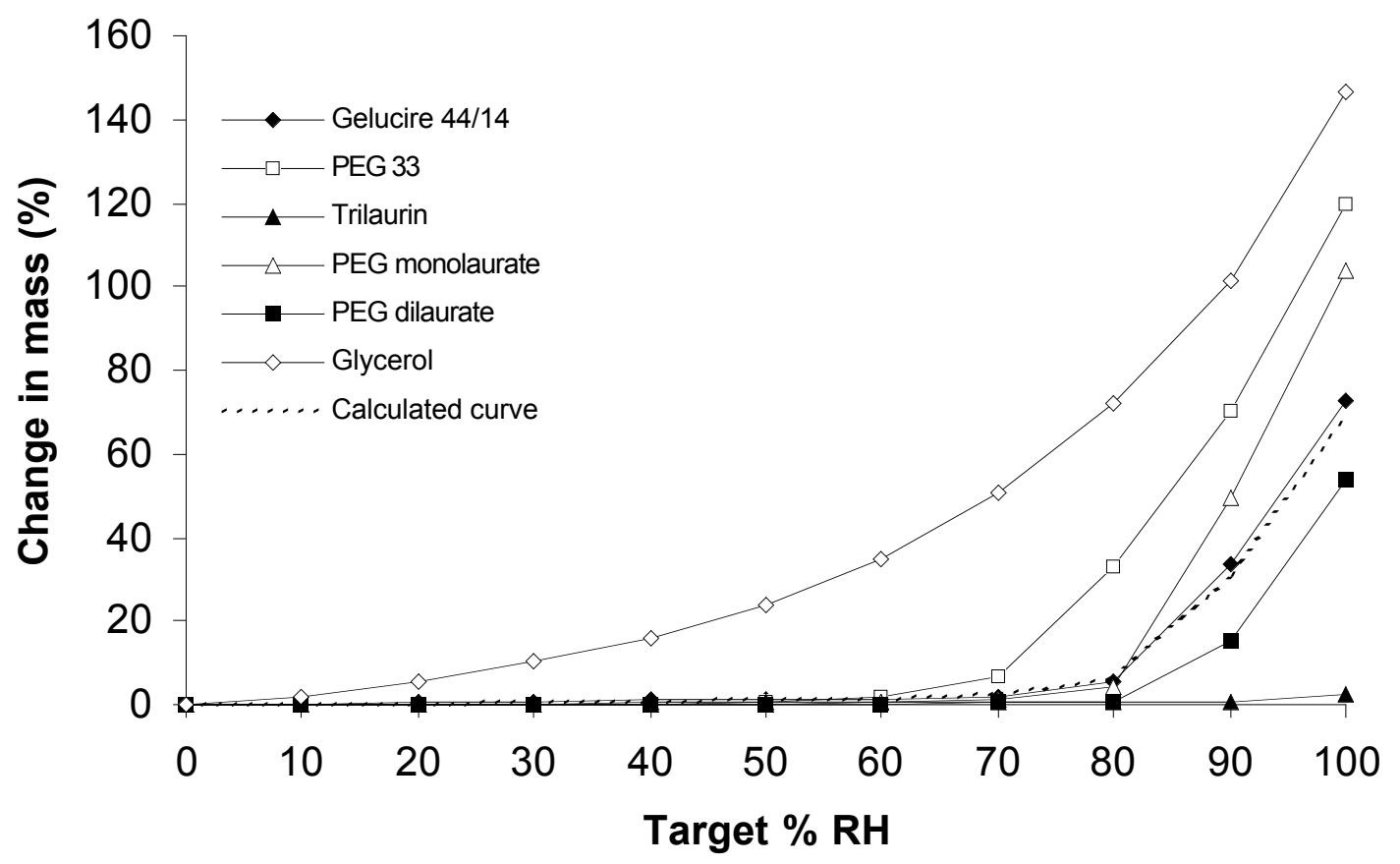

Figure 1. Dynamic water sorption isotherms for Gelucire 44/14, PEG 33, PEG monolaurate, PEG dilaurate, trilaurin. and glycerol. The calculated curve gives the change in mass as a sum of the weighted masses of the components according to their relative amounts in Gelucire 44/14 (see equation $/ 1 /$ in the text).

The water sorption isotherms of Gelucire 44/14 show a limited uptake of water at low relative humidities. For instance, at $\mathrm{RH}=50 \%$, which corresponds to ambient humidity conditions, the uptake amounts to $1 \mathrm{wt} \%$ of the mass of Gelucire 44/14. This amount is also the maximum water uptake that is allowed for Gelucire $44 / 14$ by the European Pharmacopia (Eur. Phramacopia, 2002). In this range Gelucire 44/14 is a white waxy solid.

At higher relative humidities, the uptake of water is much larger. At $\mathrm{RH}=75 \%$, TGA experiments indicate that Gelucire 44/14 contains $5 \mathrm{wt} \%$ water, but it still is a white waxy solid. At $\mathrm{RH}=84 \%$, Gelucire 44/14 contains $11 \mathrm{wt} \%$ water, and it has turned into a white dispersion. Finally, at RH $=97 \%$, Gelucire 44/14 contains $40 \mathrm{wt} \%$ water, and it has turned into a very viscous dispersion, i.e. there is actually a viscosity increase with rising humidity.

It is possible to interpret this hydration process as a sum of the hydrations of the individual components. For instance, Gelucire contains a small amount of glycerol, which adsorbs significant amounts of water, even at low RH (Figure 1). If it is assumed that glycerol constitutes $3 \mathrm{wt} \%$ of the mass of Gelucire 44/14, and that it absorbs water independently from the other components, then the uptake of water into liquid glycerol at this RH would amount to $0.7 \mathrm{wt} \%$ of the Gelucire mass. Thus, at low RH, most of the hydration of Gelucire 44/14 may be caused by water absorption into liquid glycerol. Similar results are obtained up to RH values of $68-70 \%$, 
where most of the water uptake ( $2 \mathrm{wt} \%$ of the gelucire mass) may still be caused by water absorption into glycerol.

Similarly, the faster rise of the water content of Gelucire $44 / 14$ at $\mathrm{RH}$ values above $70 \%$ is related to a surge in hydration of the other components, e.g. PEG 33 beyond $\mathrm{RH}=70 \%$ and the PEG esters beyond $\mathrm{RH}=80 \%$ (Figure 1). Over the whole range of relative humidities, the water sorption isotherm of Gelucire 44/14 can be reproduced by assuming that each component absorbed water independently. Thus, the water uptake by Gelucire, $X$, was calculated as the sum of contributions of the individual components weighted by their relative amounts $[\mathrm{xxx}]$ in Gelucire 44/14 :

$X=0.03 X_{\text {glycerol }}+0.07 X_{\text {PEG33 }}+0.71\left(0.5 X_{\text {monolaurate }}+0.5 X_{\text {dilaurate }}\right)+0.19 X_{\text {trilaurin }}$

This calculated isotherm is displayed in Figure 1. It is seen that, over the whole range of relative humidities, the calculated isotherm matches the measured water sorption isotherm of Gelucire $44 / 14$. Therefore the water uptake of Gelucire 44/14 can be reproduced by assuming that each component absorbs water independently.

In the next sections, we try to find out whether the components actually take up water independently. For this purpose, we compare the physical states of the components at each stage of the hydration process with that of the excipient.

\section{Hydration of the individual components}

\section{Glycerol}

At room temperature, glycerol is a very hygroscopic liquid (indeed, it is used in many topical formulations as a hydrating agent). The amount of water absorbed by glycerol, as a function of relative humidity, was measured by DVS (Figure 1). At $50 \% \mathrm{RH}$, the water uptake is already 24 wt $\%$ of the glycerol mass. At high RH, water sorption by glycerol still rises, but not as fast as that of the other components, and since the proportion of glycerol in the excipient is small this is no longer the main contribution to the total hydration.

\section{PEG 33}

PEG 33 is a crystalline solid at ambient conditions. WAXS experiments gave a sharp diffraction peak at a spacing of $4.6 \AA$, corresponding to the lateral distance between neighbouring chains and a small peak at $3.3 \AA$. SAXS experiments gave a weak diffraction peak at a real space repeat distance of $106.1 \AA$, corresponding to the repetition of successive lamellae (data not shown). This peak is weak because the region that separates successive lamellae has a small volume and a low contrast. A weak second order of that diffraction peak is also visible. 
In equilibrium with a humid atmosphere, the PEG crystals do not swell : DVS experiments show that, for $\mathrm{RH}<50 \%$, the water uptake is less than $0.2 \mathrm{wt} \%$ of the PEG mass. However, at RH = 50 and $60 \%$, some water uptake is measured, up to $2 \mathrm{wt} \%$ of the PEG mass (Figure 1). In the TGA experiments, the crystal - solution equilibrium was observed at $R H=75 \%$. At higher relative humidities, up to $\mathrm{RH}=90 \%$, the hydrations measured through DVS and through TGA are in good agreement with the values measured through osmotic stress (Figure 2).

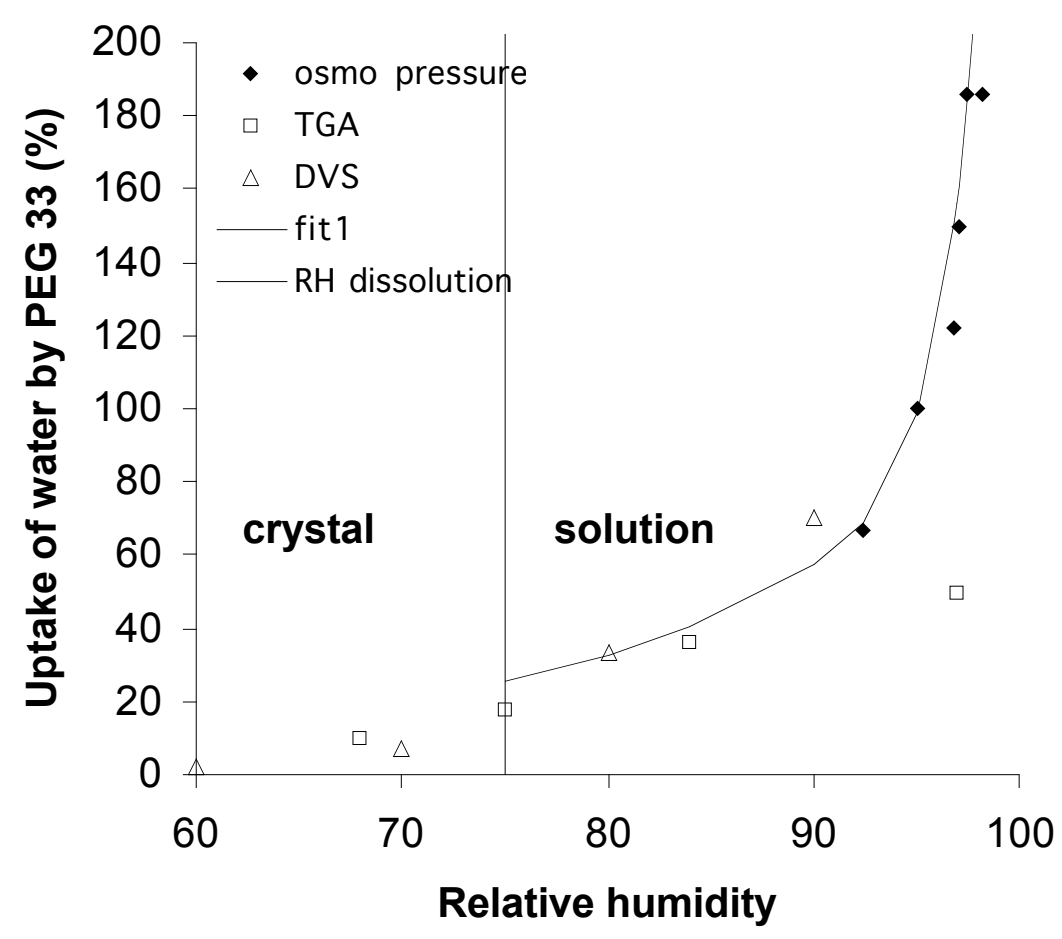

Figure 2. Equilibrium hydration of PEG 33 according to relative humidity. Filled diamonds : Osmotic pressure measurements listed by Peter Rand [yyy]. Open triangles : this work, DVS experiments. Open squares : this work, TGA experiments.

\section{$\underline{\text { PEG esters }}$}

The PEG esters also form lamellar crystals at ambient conditions. The WAXS spectra are shown in Figure 3. They are similar to the those obtained for PEG 33, with a sharp peak at $4.6 \AA$ and a small peak at $3.3 \AA$, indicating that the PEG chains have the same organization. The SAXS spectra show strong diffraction peaks at real space repeat distances of $117.1 \AA$ (for PEG 33 monolaurate) and $113.7 \AA$ (for PEG 33 dilaurate) (Figure 4). These peaks are much stronger than those of PEG 33. This indicates that the PEG lamella is sandwiched between layers that contain the alkyl chains; the strong contrast of electronic density between the PEG regions and the alkyl regions is the cause of the high intensity of the diffraction peaks. 


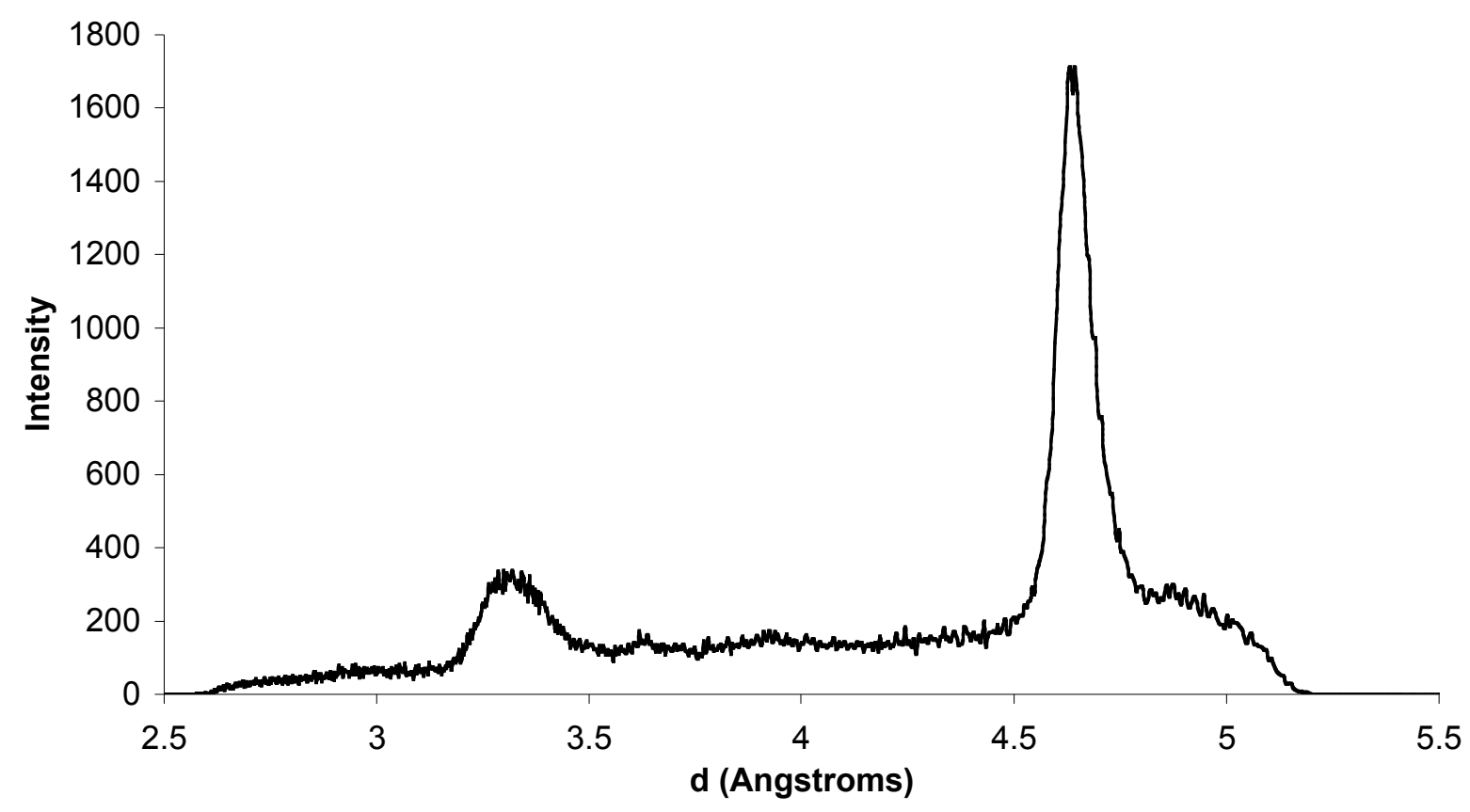

Figure 3. WAXS spectra of dry PEG dilaurate showing the peaks corresponding to the repetion of PEG chains inside a lamella.

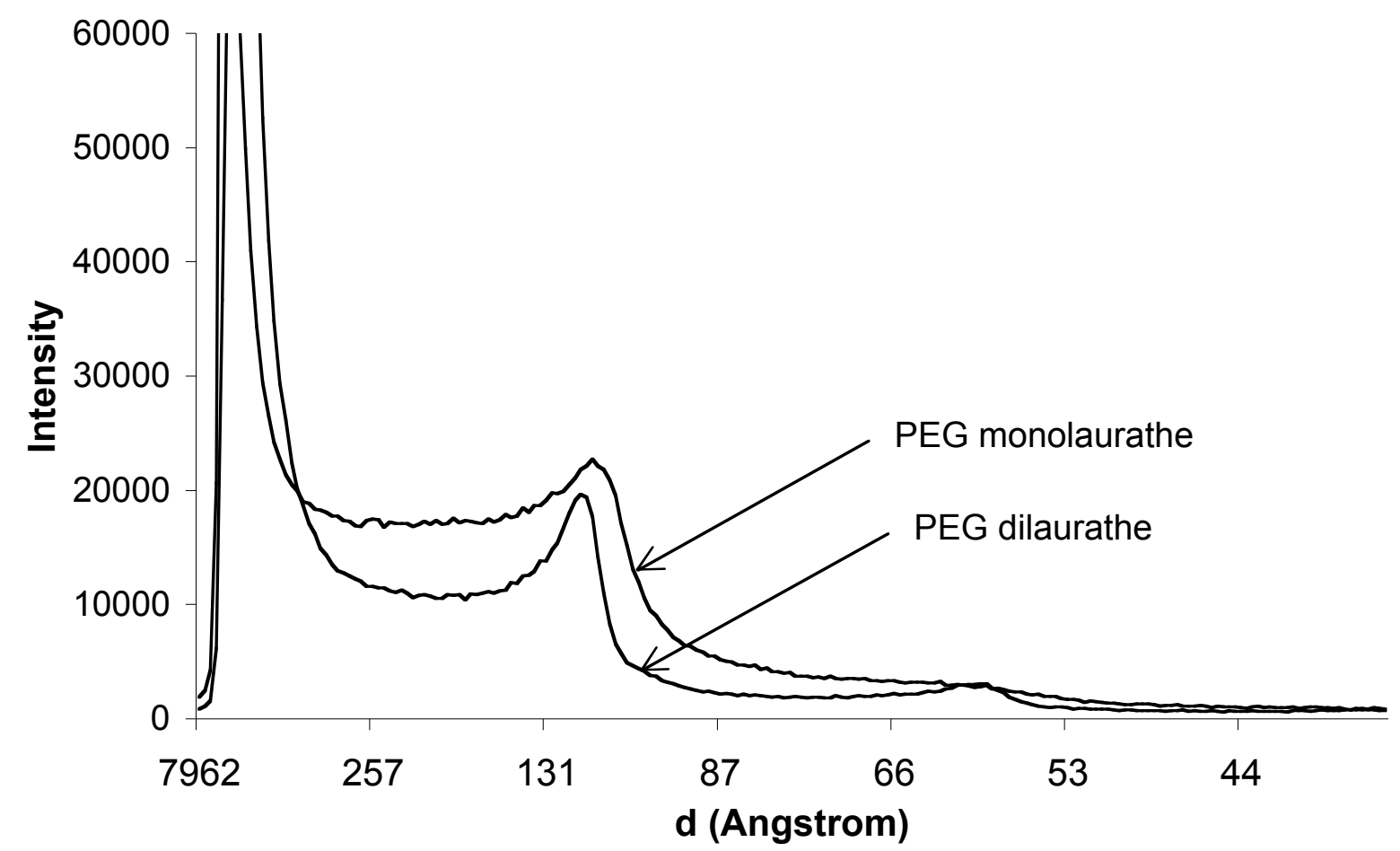

Figure 4. SAXS spectra of dry PEG esters showing the successive diffraction orders corresponding to the repetition of lamellae in a crystal. 
In equilibrium with a humid atmosphere, the crystals of PEG esters do not swell : DVS experiments show that, for all RH below $60 \%$, the water uptake is less than $0.3 \mathrm{wt} \%$ of the mass of PEG esters. Both esters begin to absorb water at RH above $80 \%$, and at $\mathrm{RH}=90 \%$ the strong water uptake indicates that dissolution is taking place. In TGA experiments performed with both esters at $\mathrm{RH}=97 \%$ we could see that all crystals had combined with water from the atmosphere to form a solution.

Other experiments were performed by mixing PEG esters with liquid water. These samples were found to form either crystals, mesophases (liquid crystals) or isotropic liquids (solutions). The phase map of the PEG monolaurate/water system is shown in Figure 5. At $20{ }^{\circ} \mathrm{C}$, samples containing less than $30 \mathrm{wt} \%$ water were mixtures of crystals with a saturated solution, as in the case of PEG 33. However, with increasing amounts of water, in the range 45-60 wt\%, a cubic mesophase was formed. This phase appears as a transparent and hard gel, and does not show birefringence when observed between crossed polarizers, i.e. it is optically isotropic. SAXS experiments performed on this cubic phase gave a set of diffraction peaks with spacings in the ratios $\sqrt{ } 4, \sqrt{ } 5, \sqrt{ } 10, \sqrt{ } 15$. The unit cell size is $a=135 \AA$ when the water content is 45 wt \% (i.e. $\mathrm{RH}=97 \%$ ) and swells with increasing water content to $143 \AA$ at $50 \mathrm{wt} \%$ water. 
(a)

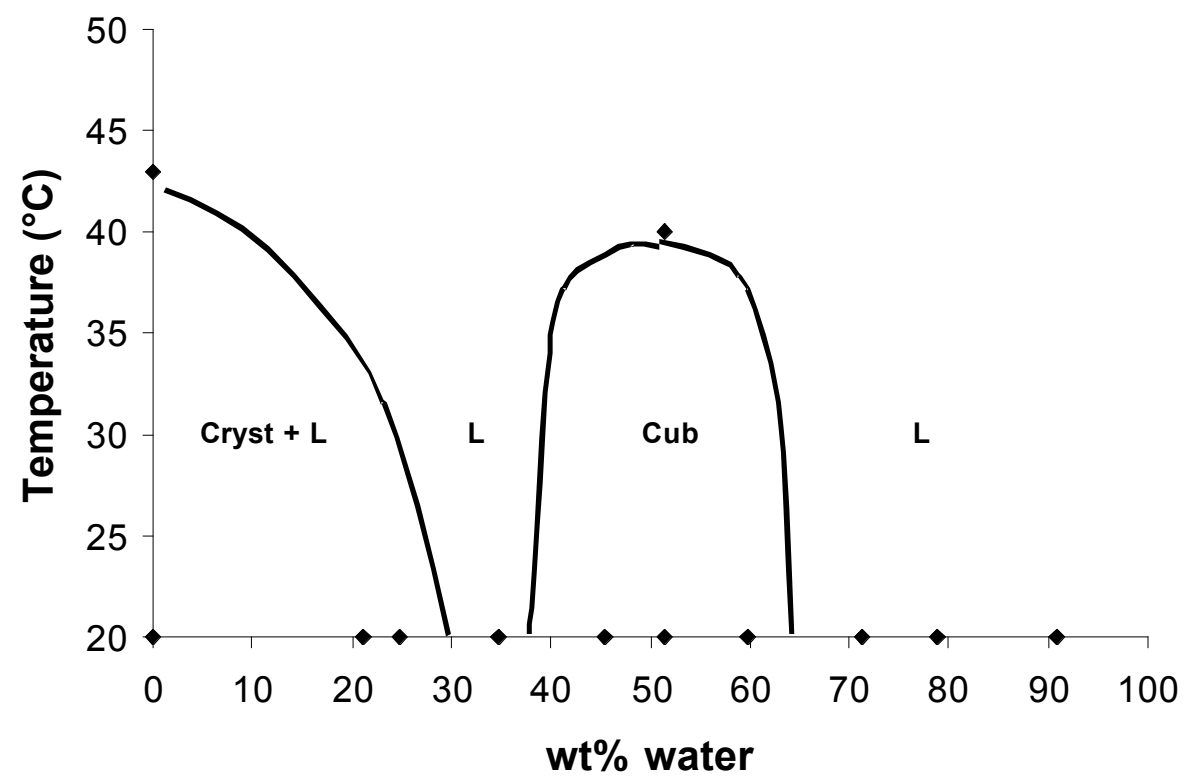

(b)

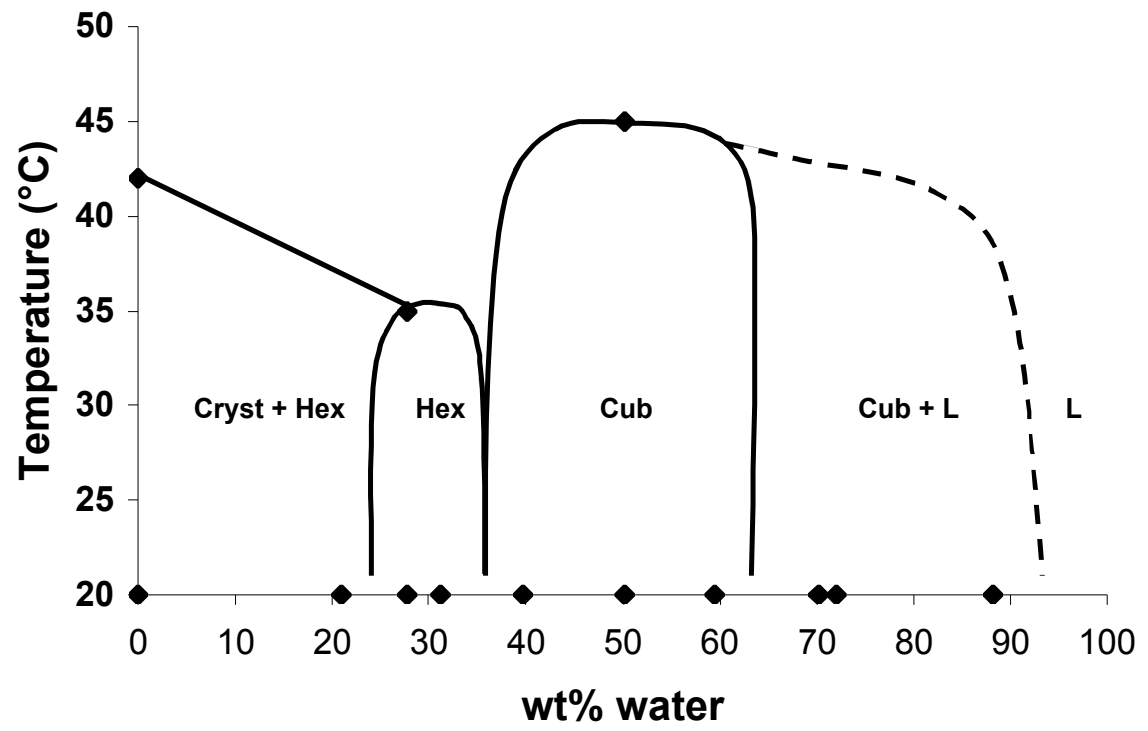

Figure 5. Phase maps of the PEG monolaurate /water (a) and PEG dilaurate /water (b) systems at temperatures $20-50{ }^{\circ} \mathrm{C}$. (Cryst. $=$ lamellar crystal, $\mathrm{L}=$ liquid solution, $\mathrm{Cub}=$ cubic mesophase, Hex = hexagonal mesophase). 
The phase map of the PEG dilaurate is also shown in Figure 5. At $20{ }^{\circ} \mathrm{C}$, samples containing less than $25 \mathrm{wt} \%$ water were mixtures of crystals with a hexagonal mesophase. The hexagonal mesophase appears as a transparent and viscous gel, and show birefringence when observed between crossed polarizers, i.e. it is optically anisotropic. The pure hexagonal mesophase was found for samples containing $27-31 \mathrm{wt} \%$ water. SAXS experiments performed on this hexagonal phase gave a set of diffraction peaks with spacings in the ratios $1, \sqrt{3}, \sqrt{ } 4$, and a unit cell size $a=62 \AA$. At higher water contents (40-60 wt\%), a cubic mesophase was found, with a unit cell size similar to that of the monolaurate $(a=130 \AA)$.

Since Gelucire contains a mixture of the PEG monolaurate and dilaurate, we also investigated the phase behaviour of mixtures containing both esters and water. The ratio in the mixture was $1 / 3$ PEG monolaurate and 2/3 PEG dilaurate as a result of the conditions during the PEG ester synthesis. It was found that the extension of the mesophases was reduced compared with the pure esters : the hexagonal phase was not formed at all, and the cubic phase was found only between 43 and $47 \mathrm{wt} \%$ water.

\section{Trilaurin}

Trilaurin is also a crystalline solid at $\mathrm{T}=20^{\circ} \mathrm{C}$. WAXS spectra of trilaurin gave the characteristic peaks of the $\beta$ form (real spacings of 4.6, 3.85, and $3.70 \AA$ ), followed by some peaks of other unidentified polymorphs. SAXS spectra gave a main peak at a real space repeat distance of $32 \AA$, corresponding to the length of 2 laurate chains.

Trilaurin is a very hydrophobic excipient. In equilibrium with a humid atmosphere, the crystals take up very little water, and this absorption is quite slow (slower than the time scales of the DVS experiments). In equilibrum with liquid water, the amount of water absorbed in the powder is only $2 \mathrm{wt} \%$ of the mass of trilaurin. Even the melted trilaurin does not mix with water to any significant extent.

\section{Hydration of Gelucire 44/14}

Dry state

The dry Gelucire $44 / 14$ is a white and waxy solid at $20^{\circ} \mathrm{C}$. According to melting enthalpies, its degree of crystallinity is $83 \%$. WAXS spectra show the same peaks as in PEG 33 and in the PEG esters, located at real space distances of 4.6 and 3.3 $\AA$. Accordingly, the PEG chains in Gelucire are crystallized. SAXS experiments show a lamellar structure with a repeat distance of 124.4 A (Figure 6). This repeat distance is close to that of the PEG esters; the small difference in repeat distance is presumably caused by a difference in the average molar mass of the PEG chains. The relative intensities of the peaks are also similar to those of the PEG esters, indicating that the content of the unit cell are quite similar. Accordingly, the crystal structure of the 
excipient must be made of PEG lamellae separated by layers of fatty acid chains, as in the PEG esters.

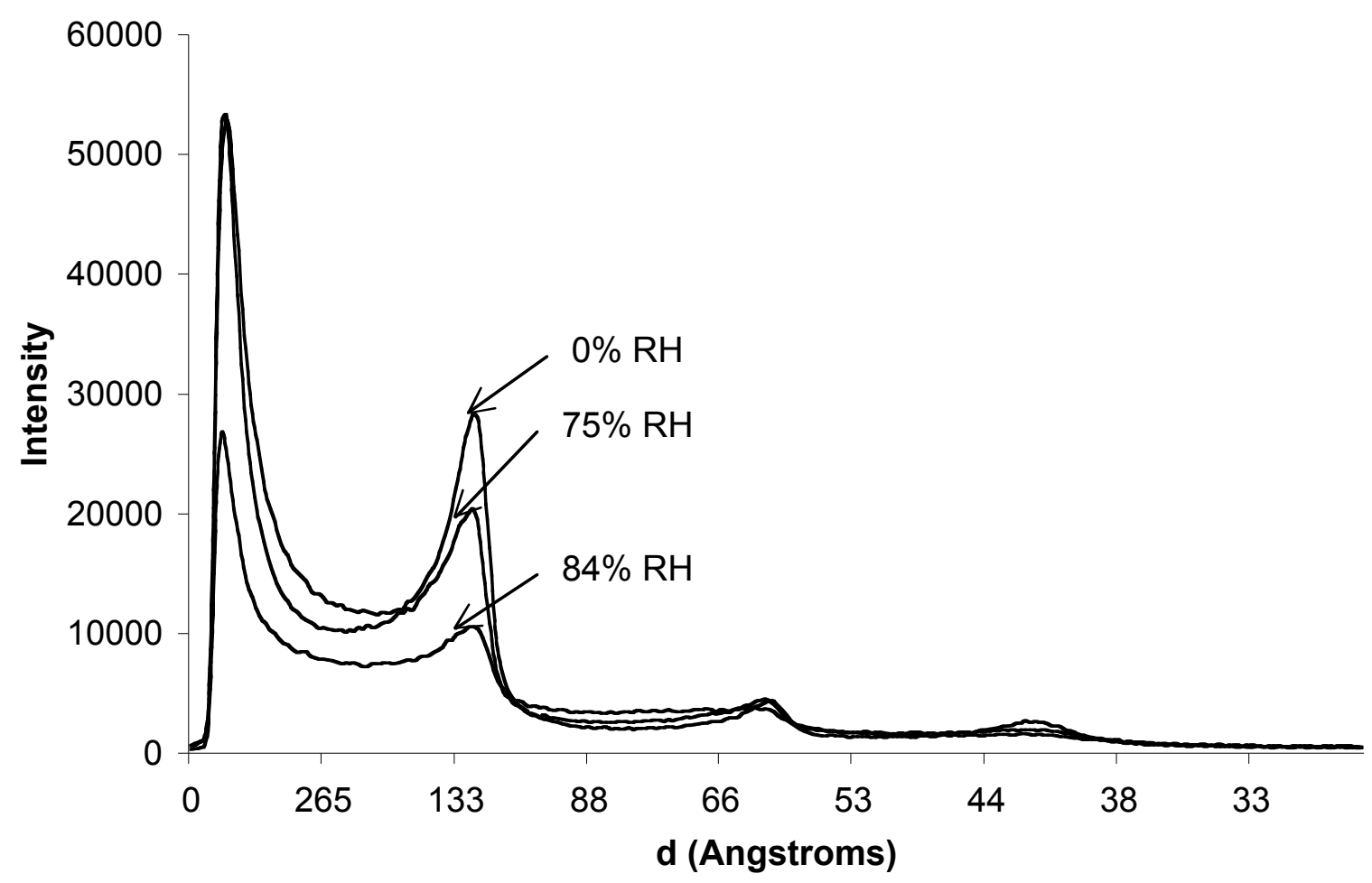

Figure 6. SAXS spectra of Gelucire 44/14 at different relative humidities. The diffraction peaks correspond to the repetition of lamellae consituted mainly of PEG esters.

\section{Hydration at ambient temperature}

At relative humidities up to $\mathrm{RH}=75 \%$, the excipient remains crystallized : indeed, its SAXS spectra are still dominated by the peaks form the PEG ester crystals (Figure 6). This is consistent with the fact that the PEG esters do not dissolve until the relative humidity exceeds 84 $\%$. The excipient remains a waxy solid, and its water content reaches $5 \mathrm{wt} \%$ at $\mathrm{RH}=75 \%$.

At $\mathrm{RH}=84 \%$, the SAXS experiment performed on the excipient shows a strong drop in the intensity of the PEG ester crystal peak. This is consistent wit the fact that the PEG 33 is fully dissolved, and PEG 33 monolaurate partly dissolved at this RH. The excipient becomes a white gel, and its water content reaches $11 \mathrm{wt} \%$.

Higher water contents were obtained by mixing the excipient with known amounts of water. These samples had the appearance of white gels (11 wt \% water), white dispersions (25-31 wt\%), white gels (50-61 wt \%), and white dispersions again (71-75 wt \%). At $90 \mathrm{wt} \%$ water, the sample separated into a white and a transparent liquid phase. 
In this range of water contents, dramatic changes were observed in the WAXS and SAXS spectra. The WAXS peaks from the crystallyzed PEG esters had vanished, indicating that the PEG chains were in a liquid state. The SAXS spectra indicated the formation of mesophases (Figure 7). At $25 \mathrm{wt} \%$ and $31 \mathrm{wt} \%$ water, a hexagonal mesophase was found. The unit cell size of this mesophase was $72 \AA$, somewhat larger than that of the pure PEG diester. At $50 \mathrm{wt} \%$ and $60 \mathrm{wt} \%$ water, a lamellar mesophase was found, with a unit cell size of $83 \AA$. This repeat distance is shorter than that of the crystallized lamellae (124.4 $\AA$ for Gelucire), which is expected since the PEG chains are now in a liquid state.

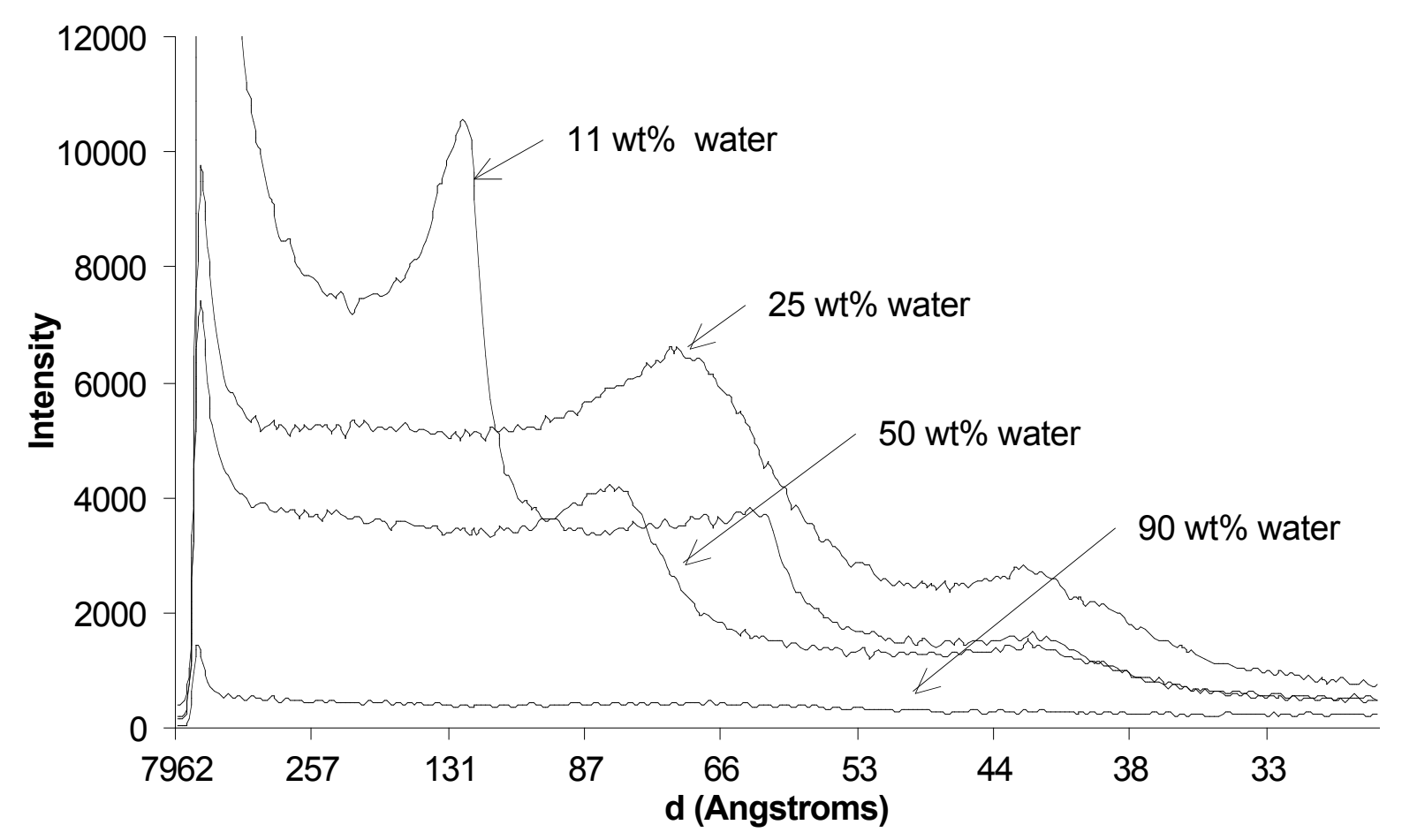

Figure 7. SAXS spectra of Gelucire 44/14 with different water contents.

Inspection of the samples using optical microscopy with crossed polarizers revealed that they contained birefringent particles (the mesophases) dispersed in a non birefringent fluid, or in a weakly birefringent fluid (for the gels formed at 50-61 wt \% water). Thus, all mesophases coexisted with another phase, which explains the white appearance of the samples.

\section{Hydration at elevated temperatures}

At higher temperatures, it was found that stronger water adsorption and dissolution take place. DSC experiments performed on samples with water content $2 \%$ gave a curve that was quite close to that of the dry material, thereby confirming that the main components (PEG esters and glycerides) were still in a solid state up to $\mathrm{T}=45^{\circ} \mathrm{C}$. However, experiments performed on samples with water content $5 \%$ showed a strong endothermal event at $30-35{ }^{\circ} \mathrm{C}$ instead of the 
fusion peak observed at $45{ }^{\circ} \mathrm{C}$ in the dry excipient. Accordingly, the combined effects of hydration $\left(5 \mathrm{wt} \%\right.$ water) and temperature $\left(35^{\circ} \mathrm{C}\right)$ cause the dissolution of the PEG esters.

A phase map of Gelucire was constructed using the results of all experiments made by mixing Gelucire 44/14 with known amounts of water (Figure 8). At low temperatures, this map shows that the dissolution of Gelucire must proceed through the formation of a set of mesophases, some of which are quite viscous (the hexagonal phase and the gel found at $50 \mathrm{wt} \%$ water). Thus, dissolution must be a slow process. On the other hand, at physiological temperatures, this map shows that all mesophases have melted : thus the dissolution becomes a fast process.

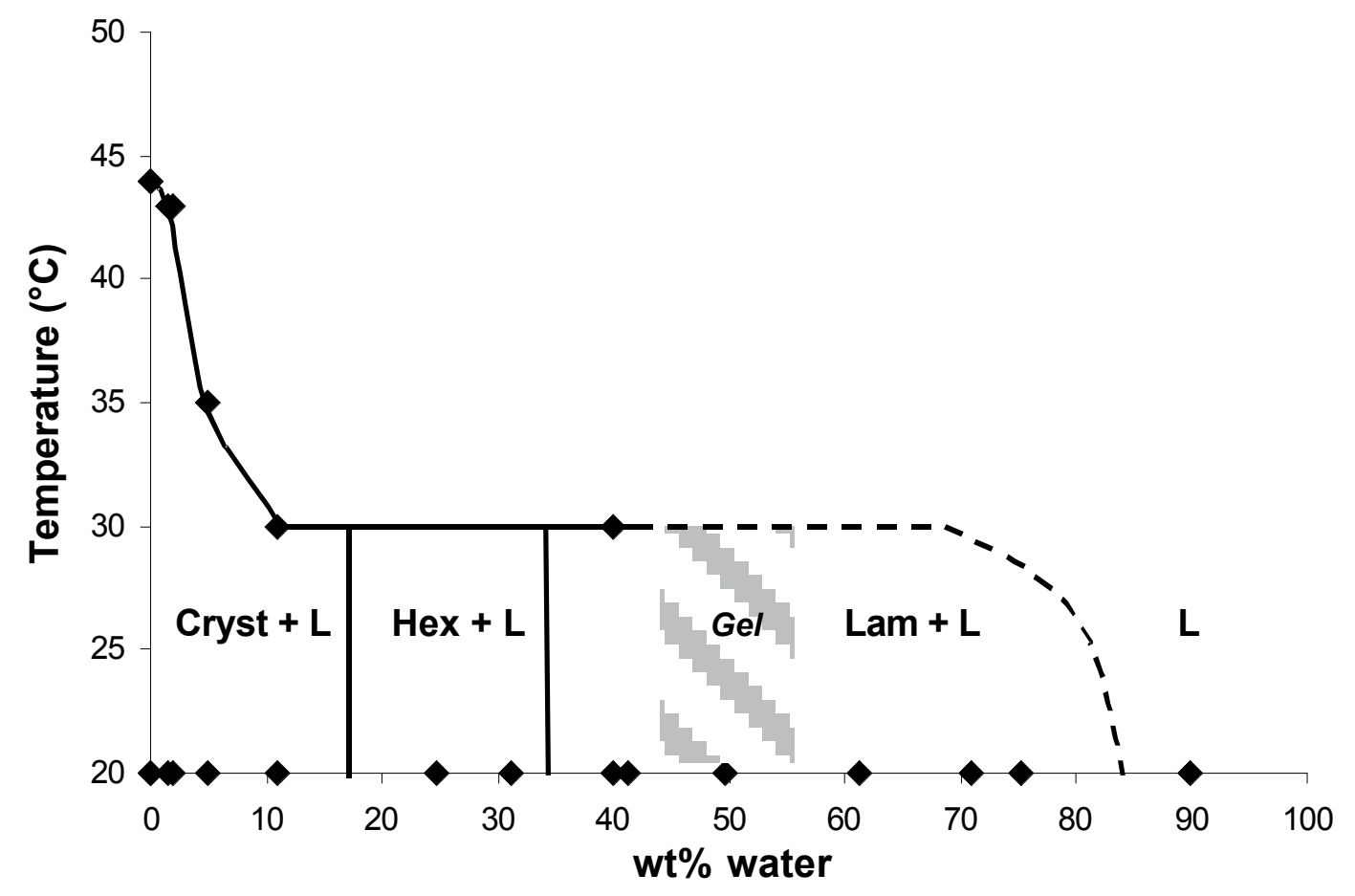

Figure 8. Phase map of Gelucire 44/14 in water at temperatures $20-50{ }^{\circ} \mathrm{C}$. (Cryst. $=$ lamellar crystal, $\mathrm{L}=$ liquid solution, Hex = hexagonal mesophase, $\mathrm{Lam}=$ lamellar mesophase). 


\section{Discussion}

\section{Dry state}

The WAXS and SAXS experiments show that Gelucire 44/14 is made mainly of lamellar crystals that contain the PEG esters and some pure PEG. In these crystals, the PEG chains form lamellae with a thickness that is determined by the length of a PEG chain in a helical configuration (bbb). These lamellae are separated by layers that contain the alkyl chains; the period of this assembly is $124 \AA$ A In addition, there may be trilaurin crystals (but they were not resolved in our x-ray experiments on dry Gelucire), amorphous regions (less than $17 \%$ of the total), and some liquid glycerol.

\section{Hydration through humid air (DVS experiments)}

The DVS and TGA experiments show that the hydration of Gelucire 44/14 follows a simple sequence. First, the most hydrophilic component, which is glycerol, absorbs water while all others remain crystalline and do not swell. The water uptake is about $1 \%$ over most of the range of RH which may be encountered during storage of the excipient $(\mathrm{RH}=30-60 \%)$. Then, at a critical RH ( $\mathrm{RH}=70 \%$ according to DVS, see the insert in Figure 1), the water uptake speeds up dramatically. This may be related to the dissolution of the next most hydrophilic component, which is PEG 33 : indeed, crystals of pure PEG 33 dissolve when the RH reaches $75 \%$, and mixtures may dissolve at a slightly lower humidity. Water is then absorbed by a solution containing glycerol and PEG 33. The other components (PEG esters and glycerides) do not swell or dissolve until the RH is quite high $(\mathrm{RH}>80 \%)$.

This sequence is exactly what would be expected for a system that contains a set of crystals in equilibrium with a humid atmosphere and with an aqueous solution: in this case, each component equilibrates separately with the aqueous solution, which is itself driven by the exchanges with the humid atmosphere. The thresholds for dissolution of the crystals may be shifted slightly with respect to those in a pure system, because the aqueous solution is a multicomponent mixture and not a 2 component solution : for instance, at $\mathrm{RH}=75 \%$, the chemical potential of PEG 33 in the solution may not be exactly that in a binary solution, because the solution actually contains glycerol as well.

\section{Hydration through mixing with liquid water}

\section{Small amounts of water}

For pharmaceutical uses, the excipient may be mixed with an active ingredient (AI). Some active ingredients contain a few water molecules per molecule of the AI. This water will then be equilibrated with all the components of the excipient, and the chemical potential of water will be 
the same throughout the mixture. Thus, the same distribution of water will be achieved as that which is obtained when the mixture is exposed to humid air, which we have measured (indeed, relative humidity also measures the chemical potential of water).

For instance, assume that the total quantity of water released by the AI amounts to $5 \mathrm{wt} \%$ of the excipient mass. This total hydration is also obtained when Gelucire is exposed to humid air at $\mathrm{RH}=75 \%$. In these conditions, our TGA measurements indicate that glycerol and PEG 33 will form a solution with water, while the other components will take up very little water (see also the DVS results shown in Figure 1). The composition of the aqueous solution will be water (33 wt $\%$ ), glycerol (20 wt $\%$ ) and PEG 33 (47 wt $\%$ ), and its volume will be $15 \mathrm{wt} \%$ of the Gelucire mass. Thus, a concentrated liquid phase may be present in the drug, dispersed in between the crystals that form the major part of the structure. If the amount of water released by the AI is less (e.g. $2 \%$ of the Gelucire mass), then only glycerol go into the aqueous solution, and the total volume of this liquid phase will be much less.

\section{Large amounts of water}

When substantial amounts of liquid water are added to the excipient, the most hydrophilic components (glycerol and PEG) dissolve immediately, and then the other crystals dissolve in this solution. However, the resulting liquids are not ordinary solutions but mesophases. In this respect, the dissolution behaviour of Gelucire differs dramatically from those of the pure components.

For the pure components, the dissolution of the crystals at room temperature or at physiological temperatures produces a succession of mesophases which are extremely viscous. In particular, both PEG esters form cubic phases at $50 \mathrm{wt} \%$ water, which appear as hard gels. These gels may take a very long time to dissolve in excess solution, because their rigid structure implies slow restructuring kinetics and long equilibration times. They do melt at high temperatures, but only when the temperature reaches $40-45{ }^{\circ} \mathrm{C}$, which is significantly above physiological temperatures.

For Gelucire 44/14, the dissolution at room temperature also produces a set of mesophases (hexagonal and lamellar). There is a viscosity maximum in the lamellar phase, which may be due to networks formed by PEG diesters connecting the lamellae. However, all these mesophases melt at temperatures below physiological temperatures. Therefore, the dissolution of Gelucire in physiological conditions may be a fast process. In this respect, the composition of Gelucire 44/14 achieves faster dissolution in water than the pure amphiphiles, e.g. the PEG diesters alone (compare Figures 5 and 8). 


\section{Conclusions}

In pharmaceutical applications, the excipient is a subject to hydration during three procedures: through mixing with a hydrated active ingredient (AI), through exposure to humid air upon storage, and through mixing with body fluids upon administration. The results presented here make it possible to make quantitative predictions for the hydration behaviour of Gelucire 44/14 in these three situations.

Exposure to humid air. In equilibrium with an atmosphere at relative humidity below $\mathrm{RH}=60$ $\%$, Gelucire takes very little water (about $1 \%$ ). However, the uptake of water becomes substantial as soon as the relative humidity exceeds $\mathrm{RH}=70 \%$. Our analysis of the hydration of the individual components shows that this uptake is almost entirely due to the hydration of the most hydrophilic components, i.e. glycerol and PEG 33. This brings two obvious conclusions : firstly, the conditions for storage of Gelucire 44/14 are obviously quite important, and secondly, a better resistance to humidity could be obtained by eliminating these hydrophilic components.

Mixtures with a hydrated AI. Many pharmaceutical active ingredients contain a few water molecules per molecule of the AI. Upon mixing with the excipient, this water will be equilibrated with all the components. Our analysis of the hydration of the individual components allows us to predict which components will dissolve, and which ones will remain crystalline. For instance, if the water added with the AI amounts to $3 \mathrm{wt} \%$ of the Gelucire mass, then the most hydrophilic components, i.e. glycerol and PEG 33 will form an aquous solution. Thus, a concentrated liquid phase may be present in the drug, dispersed in between the crystals that form the major part of the structure.

Mixtures with body fluids. At room temperature, the mixtures of Gelucire with liquid water form a set of ordered mesophases, including a viscous hexagonal phase and a highly viscous gel with lamellar ordering, which may impede further dissolution. However, at physiological temperatures, all these mesophases melt and the dissolution may be a fast process. Here as well, the comparison with the individual components is instructive : indeed, the PEG esters also form mesophases (including the very rigid cubic phases), but these mesophases do not melt at physiological temperatures, and therefore dissolution and equilibration are very slow processes. Thus, the selection of certain compositions is important because makes it possible to avoid the formation of mesophases during the dissolution in physiological conditions.

\section{References}

Craig, D.Q.M., Newton, J.M., 1991. Characterisation of polyethylene glycols using differential scanning calorimetry. Int. J. Pharm., 74, 33-41. 
Damian, F., Blaton, N., Naesens, L., Balzarini, J., Kinget, R., Augustijns, P., Van den Mooter, G., 2000. Physicochemical characterization of solid dispersions of the antiviral agent UC-781 with polyethylene glycol 6000 and Gelucire 44/14. Eur. J. Pharm. Sci., 10, 311-322.

Damian, F., Blaton, N., Kinget, R., Van den Mooter, G., 2002. Physical stability of solid dispersions of the antiviral agent UC-781 with PEG6000, Gelucire 44/14 and PVP K30. Int. J. Pharm., 244, 87-98.

Eur. Pharmacopia, $4^{\text {th }}$ Edition, 2002, Macrogols.

Gines, J.M., Veiga, M.D., Arias, M.J., Rabasco, A.M., 1995. Elaboration and thermal study of interactions between cinnarizine and gelucire 53/10 physical mixtures and solid dispersions. Int. J. Pharm., 126, 287-291.

Gupta, M.K., Goldman, D., Bogner, R.H., Tseng, Y.C., 2001. Enhanced drug dissolution and bulk properties of solid dispersions granulated with a surface adsorbent. Pharm. Dev. Technol., 6, 563-572.

Jeanmaire-Wolf, N., Segot-Chicq, S., Cohen, G., Wouessidjewe, D., 1990. Ecueil des excipients glyceridiques- Optimisation de la liberation. S.T.P. Pharma, 6, 103-109.

Mouricout, A.M., Gerbaud, D., Brossard, C., Lefort des Ylouses, D., 1990. Gélules à matrices semi-solide de gélucire - Lyodisponibilité et étude structurale. S.T.P. Pharma, 6, 368-375.

Perissutti, B., Rubessa, F., Princivalle, F., 2000. Solid dispersions of carbamazepine with Gelucire 44/14 and 50/13. S.T.P. Pharma Sci., 10, 479-484.

Roussin, P., Laforet, J-P., 1997. Investigating semi-solid formulations : Physical characterization and stability study of Gelucire registered trade mark 44/14. Fram. Vestn., 48, 260-261.

Sutananta, W., Craig, D.Q.M, Newton, J.M., 1994a. An investigation into the effect of preparation conditions on the structure and mechanical properties of pharmaceutical glyceride bases. Int. J. Pharm., 110, 75-91.

Sutananta, W., Craig, D.Q.M., Newton, J.M., 1994b. The effects of ageing on the thermal behaviour and mechanical properties of pharmaceutical glycerides. Int. J. Pharm., 111, 51-62.

xxx Only the total amount of PEG esters in Gelucire 44/14 is known. Therefore we have put $50 \%$ of each in the weighted formula.

yyy Osmotic pressures of PEG solution, private communication from Peter Rand : www.aqueous.labs.brocku.ca 